\title{
MENTOL COMO ANESTÉSICO PARA DIFERENTES CLASSES DE TAMANHO DE TILÁPIA DO NILO
}

\author{
Erivânia Gomes Teixeira', Antonio Glaydson Lima Moreira' ${ }^{1}$, Ricardo Lafaiete \\ Moreira', Francisco Roberto dos Santos Lima ${ }^{1}$
}

\author{
${ }^{1}$ Universidade Federal do Ceará \\ Endereço para correspondência: Erivânia Gomes Teixeira: vaniaengp@yahoo.com.br
}

\begin{abstract}
RESUMO: O objetivo com o trabalho foi avaliar o mentol como anestésico para diferentes classes de tamanho de tilápia do Nilo. Foram avaliadas três classes de tamanho: alevinos, juvenis e adultos, submetidas a seis concentrações de mentol $(30,60,120,180,240$ e $300 \mathrm{mg} \mathrm{L}-1)$, expostos individualmente por 10 minutos à solução anestésica e acompanhados durante a indução e recuperação. Para os alevinos, juvenis e adultos as concentrações recomendadas para atingirem o estágio de anestesia profunda foram de $60(124,8 \pm 6,9 \mathrm{~s}), 180(202.7 \pm 17.0 \mathrm{~s})$ e $240(210,5 \pm 17,3 \mathrm{~s})$ $\mathrm{mg} \mathrm{L-1}$, respectivamente. Os tempos de recuperação para as concentrações supracitadas foram, na mesma ordem, de 306,2 $\pm 27,1,202,7 \pm 17,0$ e 328,6 $\pm 32,0$ s. Para indução ao estágio de anestesia cirúrgica, as concentrações foram de 60 (172,8 $\pm 10,1 \mathrm{~s}), 120$ (313,7 $\pm 14,7 \mathrm{~s})$ para alevinos e juvenis, respectivamente. A utilização do mentol como anestésico mostra-se eficaz, variando entre as diferentes classes de tamanho de tilápia do Nilo.
\end{abstract}

Palavras-chave: aqüicultura; anestesia; estresse.

\section{MENTHOL AS AN ANESTHETIC FOR DIFFERENT SIZE CLASSES OF NILE TILAPIA}

\begin{abstract}
The aim of this study was to evaluate the menthol as an anesthetic for various size classes of Nile tilapia. We evaluated three size classes: fingerlings, juveniles and adults, subjected to six concentrations of menthol $(30,60,120,180,240$ and $300 \mathrm{mg} \mathrm{L}-1)$, individually exposed for 10 minutes to the anesthetic solution and monitored during the induction and recovery. To fingerlings, juveniles and adults the recommended concentrations to reach the stage of deep anesthesia were 60 $(124,8 \pm 6,9 \mathrm{~s}), 180(202,7 \pm 17,0 \mathrm{~s})$ and $240(210,5 \pm 17,3 \mathrm{~s}) \mathrm{mg} \mathrm{L}-1$, respectively. The recovery times for the concentrations above were in the same order, from 306,2 $\pm 27,1,202,7 \pm 17,0$, and $328,6 \pm 32,0 \mathrm{~s}$. For the induction stage of surgical anesthesia, the concentrations were $60(172,8 \pm$ $10,1 \mathrm{~s}), 120(313,7 \pm 14,7 \mathrm{~s})$ for fingerlings and juveniles, respectively. The use of menthol as an anesthetic is effective, varying between the different size classes of Nile tilapia.
\end{abstract}

Key Words: aquaculture; anesthetic; stress. 


\section{INTRODUÇÃO}

Peixes submetidos às práticas intensas de manejo como o transporte, adensamento, mudanças na qualidade da água, temperatura e salinidade estão sujeitos a uma série de fatores estressantes (Vidal et al., 2008). Diversas substâncias, com propriedades diferentes, são frequentemente utilizadas como anestésico para peixes com o objetivo de reduzir o estresse e facilitar o manejo (Façanha e Gomes, 2005; Ross e Ross, 2008).

A eficácia das substâncias anestésicas varia intra e inter espécies. Peixes com tamanho, idade e sexo diferentes podem responder de forma contrária a uma concentração em particular, estas respostas também podem ser influenciadas por fatores tais como $\mathrm{pH}$, salinidade, temperatura $\mathrm{e}$ nível de oxigênio dissolvido na água (Park et al, 2008; Zahl et al., 2009). Diferentes anestésicos exigem concentrações diferentes para atingir o estágio de anestesia desejado. $\mathrm{O}$ anestésico adequado deve induzir à anestesia em até três minutos e recuperação em menos de dez minutos (Park et al., 2003), proporcionar segurança para os mamíferos, ter custo acessível, deixar pouco resíduo após período de depuração de uma hora ou menos e não apresentar toxicidade aos peixes (Marking e Meyer, 1985). Estudos realizados por Façanha e Gomes (2005), indicaram que quando o peixe é exposto à concentração ideal de mentol não ocorrem distúrbios fisiológicos de glicose plasmática e no $\mathrm{Na}+$ plasmático, no entanto, outras pesquisas devem ser realizadas com a finalidade de verificar a toxicidade e o resíduo presente nos espécimes submetidos a este anestésico.

O mentol é um óleo essencial extraído de plantas do gênero Mentha (Patel et al., 2007), com conhecida propriedade anestésica (Rupert e
Barnes, 1994) e antiflamatória (Lorenzo et al., 2002). Os estudos sobre o uso de substâncias naturais, como o mentol (Façanha e Gomes, 2005; Gonçalves et al., 2008) e o eugenol (Vidal et al., 2008; Weber et al., 2009) como anestésico em aqüicultura tem origem na necessidade de se encontrar produtos alternativos eficazes em substituição aos anestésicos sintéticos legalmente utilizados. Estas substâncias naturais, geralmente apresentam baixo custo e fácil aquisição (Roubach et al., 2005), justificando assim o desenvolvimento de protocolos adequados para sua utilização em peixes.

A tilápia do Nilo (Oreochromis niloticus) é uma das espécies mais importante para a aqüicultura mundial e atualmente é a espécie mais cultivada na piscicultura brasileira (FAO, 2007). A utilização de anestésicos durante 0 manejo das diferentes classes de tamanho de tilápia é justificada para facilitar 0 manuseio de espécimes adultos durante procedimentos laboratoriais e de campo tendo em vista que a espécie caracteriza-se pela existência de espinhos ósseos na nadadeira dorsal e pélvica que podem provocar dolorosas lesões nos técnicos. Para alevinos e juvenis o uso de anestésico justifica-se, principalmente, durante o transporte, pois a utilização de baixas concentrações possibilita a redução do estresse e/ou mortalidade. Além disso, tilápias de tamanhos diversos, exigem tempos de indução anestésica distintos (MOREIRA et al., 2010) e, consequentemente, exigem concentrações diferentes para atingir o mesmo estágio de anestesia em tempo considerado seguro.

Portanto, o objetivo deste trabalho foi avaliar o mentol como anestésico e determinar a concentração recomendada para o manejo de diferentes classes de tamanho de tilápia do Nilo 


\section{MATERIAL E MÉTODOS}

Foram

realizados

três experimentos, no período de dezembro de 2008 a março de 2009, na Estação de Piscicultura pertencente ao Departamento de Engenharia de Pesca, da Universidade Federal do Ceará e no Centro de Pesquisa em Aqüicultura do Departamento Nacional de Obras Contra as Secas (Pentecoste, Ceará) onde os peixes foram adquiridos.

Foram utilizadas três diferentes classes, com 72 espécimes de tilápia do Nilo, cada grupo. O peso médio dos indivíduos utilizados foi de $1,18 \pm 0,3 \mathrm{~g}$, $28,3 \pm 6,6$ g e $660,0 \pm 91,5 \mathrm{~g}$ para alevinos, juvenis e adultos, respectivamente. Para aclimatação, os animais foram estocados em tanques, providos de aeração constante. Durante cinco dias os peixes foram alimentados com ração comercial adequada a cada fase do ciclo de vida. Todos os indivíduos obedeceram a um jejum de 24 horas antes da realização dos experimentos e foram avaliados diariamente quanto à atividade, aparência externa e alimentação.

A exposição ao anestésico dos alevinos, juvenis e adultos, foi conduzida em aquários de vidro com volumes úteis de 2,3 e $60 \mathrm{~L}$, respectivamente. Todos os experimentos foram providos de aeração constante, mantida através de pedra porosa acoplada a um soprador. A temperatura, oxigênio e $\mathrm{pH}$ foram mantidos dentro dos parâmetros recomendados para a espécie de acordo com Vinatea (1997). Foi utilizado o mentol $\left(\mathrm{C}_{10} \mathrm{H}_{20} \mathrm{O}\right)$ puríssimo, em forma de cristal, da marca Vetec $\AA$, que foi diluído em álcool etílico $\mathrm{P}$. A. resultando em uma solução estoque à concentração de $100 \mathrm{mg} \mathrm{mL}^{-1}$. Para se determinar a ação do mentol sobre a indução e recuperação anestésica dos animais, foram testadas seis concentrações anestésicas (30, 60, 120,
$180, \quad 240$ e $\left.300 \quad \mathrm{mg} \mathrm{L} \mathrm{L}^{-1}\right) . \quad$ As concentrações testadas foram estabelecidas a partir de resultados obtidos para outras espécies (Gonçalves et al., 2008; Façanha e Gomes, 2005). Para cada tratamento, doze peixes foram submetidos, individualmente, ao banho anestésico por 10 minutos. Durante a imersão, foi registrado o tempo para atingir cada estágio de anestesia, conforme descrito por Ross e Ross (2008) de acordo com a tabela 1. A ausência de reação aos estímulos foi verificada pelo toque na lateral dos peixes com um bastão de vidro e a perda do tônus muscular foi considerada quando, ao ser retirado da água e colocado em posição lateral, a cauda do peixe permanecia totalmente pendida para baixo (VIDAL et al., 2008).

Tabela 1 - Estágios de anestesia em peixes.

\begin{tabular}{|c|c|c|}
\hline Está & & Resposta comportamental em peixes \\
\hline I & $\begin{array}{l}\text { Sedação } \\
\text { leve }\end{array}$ & $\begin{array}{l}\text { Reativos a estímulos externos; } \\
\text { movimentos reduzidos, batimentos } \\
\text { operculares mais lentos; equilíbrio } \\
\text { normal. }\end{array}$ \\
\hline II & $\begin{array}{l}\text { Sedação } \\
\text { profunda }\end{array}$ & $\begin{array}{l}\text { Perda total da reatividade aos } \\
\text { estímulos externos, exceto forte } \\
\text { pressão; leve queda do movimento } \\
\text { opercular; equilíbrio normal. }\end{array}$ \\
\hline III & Narcose & $\begin{array}{l}\text { Perda parcial do tonus muscular; } \\
\text { natação errática, aumento dos } \\
\text { movimentos operculares; reativos } \\
\text { apenas a forte estímulo tátil ou } \\
\text { vibração. }\end{array}$ \\
\hline IV & $\begin{array}{l}\text { Anestesia } \\
\text { profunda }\end{array}$ & $\begin{array}{l}\text { Perda total de tônus muscular; perda } \\
\text { total de equilíbrio; batimento } \\
\text { opercular lento, porém regular. }\end{array}$ \\
\hline V & $\begin{array}{l}\text { Anestesia } \\
\text { cirúrgica }\end{array}$ & $\begin{array}{l}\text { Ausência total de reação, mesmo a } \\
\text { forte estímulo; movimentos } \\
\text { operculares lentos e irregulares; } \\
\text { batimentos cardíacos lentos; perda } \\
\text { total de todos os reflexos. }\end{array}$ \\
\hline VI & $\begin{array}{l}\text { Colapso } \\
\text { medular }\end{array}$ & $\begin{array}{l}\text { Parada da ventilação; parada } \\
\text { cardíaca; morte eventual. }\end{array}$ \\
\hline
\end{tabular}

Ao final dos 10 minutos, os peixes foram retirados do aquário de indução e transferidos para o aquário de recuperação, com volume similar aos de indução, contendo água sem mentol e provido de aeração. O indivíduo foi considerado recuperado quando retomou totalmente seu equilíbrio e capacidade natatória, de acordo com Vidal et al., (2008). Os tempos de indução aos diferentes estágios de 
anestesia e de recuperação foram aferidos através da utilização de cronômetro digital.

Os resultados obtidos foram submetidos a análises de variância (ANOVA), e comparação de médias realizadas pelo software BIOESTAT versão 5.0, utilizando o teste de Tukey a $5 \%$ de significância.

\section{RESULTADOS E DISCUSSÃO}

Os peixes utilizados no experimento apresentaram atividade e aparência externa normais indicando boa sanidade, ratificada pela ausência de mortalidade durante 0 tempo de aclimatação. Também não foi observado nenhuma mortalidade ou efeito adverso dentro de 24 horas após o procedimento experimental. Inoue et al. (2003), utilizando óleo de cravo como anestésico para juvenis de matrinxã, também não registrou mortalidade em período de observação semelhante.

Durante a indução anestésica foi observada a passagem seqüencial pelos diversos estágios conferidos pelos seguintes padrões comportamentais: redução dos movimentos, batimento opercular lento, perda de reação a estímulos externos, natação errática, perda do tônus muscular, ausência total de reação e movimentos operculares lentos e irregulares. Estes padrões comportamentais produzidos por anestésicos em peixes foram descritos por Ross e Ross (2008) e observados por Façanha e Gomes (2005) utilizando mentol como anestésico para o tambaqui, Colossoma macropomum.

Conforme a tabela 2, para os alevinos, todas as concentrações testadas permitiram a passagem pela seqüência de estágios anestésicos, até atingirem a fase de anestesia profunda, recomendada para procedimentos de manejo. Vidal et al. (2008), empregando eugenol para indução anestésica de tilápia, relataram que a passagem gradativa pela seqüência de estágios não foi evidenciada nas maiores concentrações testadas devido à velocidade de ação do anestésico, enquanto Mylonas et al. (2005) observaram a progressiva sequência dos estágios anestésicos alcançadas por Dicentrarchus labrax e Sparus aurata anestesiados com eugenol e 2fenoxietanol.

Tabela 2 - Eventos comportamentais (em segundos) de alevinos de tilápia do Nilo expostos a diferentes concentrações de mentol ${ }^{(1)}$.

\begin{tabular}{|c|c|c|c|c|c|c|}
\hline \multirow{2}{*}{$\begin{array}{l}\text { Concent } \\
\text { ração } \\
\left(\mathrm{mg} \mathrm{L}^{-1}\right)\end{array}$} & \multicolumn{6}{|c|}{ Evento comportamental (segundos) } \\
\hline & $\begin{array}{l}\text { Está } \\
\text { gio I }\end{array}$ & $\begin{array}{l}\text { Estág } \\
\text { io II }\end{array}$ & $\begin{array}{l}\text { Estágic } \\
\text { III }\end{array}$ & $\begin{array}{l}\text { Estági } \\
\text { o IV }\end{array}$ & $\begin{array}{l}\text { Estág } \\
\text { io V }\end{array}$ & $\begin{array}{l}\text { Recup } \\
\text { eração }\end{array}$ \\
\hline 30 & $\begin{array}{l}121, \\
8 \pm \\
13,1^{ \pm} \\
50,8\end{array}$ & $\begin{array}{l}203,0 \\
\pm \\
15,3^{a}\end{array}$ & $\begin{array}{l}301,7 \\
\pm \\
19,7^{a}\end{array}$ & $\begin{array}{l}466,0 \\
\pm \\
30,8^{a}\end{array}$ & 172,8 & $\begin{array}{l}118,0 \pm \\
20,5^{\mathrm{a}}\end{array}$ \\
\hline 60 & $\begin{array}{l} \pm \\
1,9^{b} \\
32,2\end{array}$ & $\begin{array}{l}74,8 \pm \\
5,0^{b}\end{array}$ & $\begin{array}{l}92,2 \\
5,1^{b}\end{array}$ & $\begin{array}{l}124,8 \\
\pm 6,9^{b}\end{array}$ & $\frac{ \pm}{10,1^{\mathrm{a}}}$ & $\begin{array}{l}306,2 \pm \\
27,1^{a}, b\end{array}$ \\
\hline 120 & $\begin{array}{l} \pm 2,4^{b} \\
23,3\end{array}$ & $\begin{array}{l}45,5 \pm \\
3,2^{c}\end{array}$ & $\begin{array}{l}57,6 \pm \\
3,1^{\mathrm{b}, \mathrm{c}}\end{array}$ & $\begin{array}{l}79,6 \\
2,5^{c}\end{array}$ & $\begin{array}{l}100,7 \\
\pm 3,6^{b}\end{array}$ & $\begin{array}{l}360,6 \pm \\
32,0^{a, b}\end{array}$ \\
\hline 180 & $\begin{array}{l} \pm \\
2,3^{c} \\
16,8\end{array}$ & $\begin{array}{l}31,8 \pm \\
2,7^{c}\end{array}$ & $\begin{array}{l}40,4 \pm \\
3,0^{c}\end{array}$ & $\begin{array}{l}51,0 \\
2,6^{c, d}\end{array}$ & $\begin{array}{l}64,2 \pm \\
3,0^{c}\end{array}$ & $\begin{array}{l}690,7 \pm \\
117,8^{c}\end{array}$ \\
\hline 240 & $\begin{array}{l} \pm \\
1,0^{c} \\
11,9\end{array}$ & $\begin{array}{l}23,9 \pm \\
1,3^{c}\end{array}$ & $\begin{array}{l}31,2 \pm \\
1,4^{\mathrm{C}}\end{array}$ & $\begin{array}{l}42,1 \\
2,2^{d}\end{array} \pm$ & $\begin{array}{l}56,8 \pm \\
3,4^{c}\end{array}$ & $\begin{array}{l}583,2 \pm \\
104,0^{b, c}\end{array}$ \\
\hline 300 & $\pm^{ \pm}, 7^{c}$ & $\begin{array}{l}19,8 \pm \\
1,0^{c}\end{array}$ & $\begin{array}{l}28,7 \pm \\
1,1^{\mathrm{c}}\end{array}$ & $\begin{array}{l}40,6 \pm \\
1,5^{d}\end{array}$ & $\begin{array}{l}54, \quad \pm \\
2,9^{c}\end{array}$ & $\begin{array}{l}899,2 \pm \pm \\
148,8^{c}\end{array}$ \\
\hline
\end{tabular}

* Estágio não alcançado. (1) Médias seguidas pela mesma letra em coluna não diferem entre si pelo teste de Tukey $(\mathrm{P}>0.05)$.

No presente trabalho os resultados mostraram que a partir da concentração de $60 \mathrm{mg} \mathrm{L}^{-1}$ os alevinos foram induzidos à anestesia profunda e cirúrgica em tempo inferior a 3 minutos, intervalo de tempo considerado seguro segundo Park et al. (2003). Desta forma, a concentração recomendada para os animais atingirem estes estágios é de $60 \mathrm{mg} \mathrm{L}^{-1}$ (Tabela 2). Esta mesma concentração foi indicada por Barbosa et al. (2007) utilizando eugenol como anestésico para matrinxã. A referida concentração permitiu uma anestesia profunda em $124,8 \pm 6,9 \mathrm{~s}$, tempo considerado dentro da faixa ideal para procedimentos de manejo. 
No presente trabalho, a anestesia cirúrgica foi atingida em $172,8 \pm 10,1 \mathrm{~s}$ e a recuperação em $306,2 \pm 27,1$ s para alevinos. Este resultado foi semelhante ao aconselhado por Marking e Meyer (1985) para diferentes espécies de peixes. De acordo com Park et al. (2003), uma das características desejáveis em um anestésico é a capacidade de induzir à anestesia em 3 minutos e possibilitar a recuperação dentro de 10 minutos.

Em relação aos juvenis (tabela 3 ), a concentração de $30 \mathrm{mg} \mathrm{L}^{-1}$ não foi suficiente para induzir, durante exposição de 10 minutos, ao estágio de anestesia profunda, revelando que estes foram mais resistentes ao procedimento quando comparados com os alevinos. King et al. (2005) afirmaram que a eficácia e a segurança de qualquer anestésico variam entre espécies, estágio de desenvolvimento e condições ambientais.

Tabela 3 - Eventos comportamentais (em segundos) de juvenis de tilápia do Nilo, expostos a diferentes concentrações de mentol ${ }^{(1)}$

\begin{tabular}{|c|c|c|c|c|c|c|}
\hline \multirow{2}{*}{$\begin{array}{l}\text { Concent } \\
\text { ração } \\
\left(\mathrm{mg} \mathrm{L}^{-1}\right)\end{array}$} & \multicolumn{6}{|c|}{ Evento comportamental (segundos) } \\
\hline & $\begin{array}{l}\text { Está } \\
\text { gio I }\end{array}$ & $\begin{array}{l}\text { Estági } \\
\text { o II }\end{array}$ & $\begin{array}{l}\text { Estági } \\
\text { o III }\end{array}$ & $\begin{array}{l}\text { Estági } \\
\text { o IV }\end{array}$ & $\begin{array}{l}\text { Estági } \\
\text { o V }\end{array}$ & $\begin{array}{l}\text { Recup } \\
\text { eração }\end{array}$ \\
\hline & $\begin{array}{l}80,4 \\
\pm\end{array}$ & $\begin{array}{l}237,5 \\
\pm\end{array}$ & $\begin{array}{l}415,8 \\
\pm\end{array}$ & & & \\
\hline 30 & $\begin{array}{l}\overline{8}, 1^{a} \\
60,4\end{array}$ & $\overline{24,1^{\mathrm{a}}}$ & $24,0^{\mathrm{a}}$ & * & * & * \\
\hline 60 & $\underset{5}{ \pm}, 9^{a}$ & $\begin{array}{l}124,9 \\
\pm 7,0^{b}\end{array}$ & $\begin{array}{l}203,0 \\
\pm \\
14,1^{b}\end{array}$ & $\begin{array}{l}306,8 \\
\pm 39,4^{\mathrm{a}}\end{array}$ & $\begin{array}{l}436,7 \\
\pm \\
29,2^{\mathrm{a}}\end{array}$ & $\begin{array}{l}248,0 \\
\pm 16,7^{\mathrm{a}}\end{array}$ \\
\hline & $\begin{array}{l}50,9 \\
\pm\end{array}$ & & & & & 317,5 \\
\hline 120 & $\begin{array}{l}5,0^{b}, \\
36,6\end{array}$ & $\begin{array}{l}92,7 \pm \\
6,1^{b, c}\end{array}$ & $\begin{array}{l}139,8 \\
\pm 7,1^{c} \\
1316\end{array}$ & $\begin{array}{l}218,7 \\
\pm 16,0^{b}\end{array}$ & $\begin{array}{l} \pm \\
14,7^{\mathrm{b}}\end{array}$ & \pm \\
\hline 180 & $\begin{array}{l}5,0 \\
\pm \\
1,8^{c} \\
44,1\end{array}$ & $\begin{array}{l}80,7 \pm \\
7,4^{\mathrm{c}}\end{array}$ & $\begin{array}{l} \pm \\
10,7^{c}\end{array}$ & $\begin{array}{l} \pm \\
\pm \\
17,0^{\mathrm{b}, \mathrm{c}}\end{array}$ & $\begin{array}{l} \pm \\
18,9^{b}\end{array}$ & $\begin{array}{l} \pm \\
25,2^{b, c}\end{array}$ \\
\hline 240 & ${ }_{c}^{ \pm}, 8^{b}$ & $\begin{array}{l}80,1 \pm \\
4,1^{c}\end{array}$ & $\begin{array}{l}127,6 \\
\pm 9,2^{\mathrm{c}}\end{array}$ & $\begin{array}{l}196,5 \\
\pm \\
10,3^{\mathrm{b}, \mathrm{c}}\end{array}$ & $\begin{array}{l}311,9 \\
\pm \\
17,6^{b}\end{array}$ & $\begin{array}{l}313,6 \\
\pm \\
17,4^{\mathrm{a}, \mathrm{b}}\end{array}$ \\
\hline 300 & $\begin{array}{l}35,2 \\
\pm \\
2,9^{c}\end{array}$ & $\begin{array}{l}63,2 \pm \\
4,2^{\mathrm{c}}\end{array}$ & $\begin{array}{l}99,2 \pm \\
4,4^{c}\end{array}$ & $\begin{array}{r}153,3 \\
\pm 8,4^{\mathrm{c}} \\
\end{array}$ & $\begin{array}{l}305,6 \\
\pm \\
14,4^{\mathrm{b}}\end{array}$ & $\begin{array}{l}406,2 \\
\pm 24,9^{c} \\
\end{array}$ \\
\hline
\end{tabular}

Os juvenis expostos às concentrações de 60 a $300 \mathrm{mg} \mathrm{L}^{-1}$ foram induzidos a todos os estágios de anestesia, no entanto em relação ao tempo de indução à anestesia profunda, as concentrações de 120, 180 e 240 mg $\mathrm{L}^{-1}$ induziram a este estágio em $218,7 \pm 16,0$ s, $202,7 \pm \quad 17,0 s \quad$ e $196,5 \pm 10,3 \mathrm{~s}$ respectivamente, sem diferença estatisticamente significativa entre as mesmas. De acordo com os resultados obtidos, a concentração recomendada para anestesia voltada para biometria e breve manejo é a de $180 \mathrm{mg} \mathrm{L}^{-1}$, tendo em vista que a mesma apresentou tempo ideal de indução em 202,7 \pm 17,0s e recuperação em $378,7 \pm 25,2$ s, dentro dos limites de segurança.

Estes resultados foram diferentes dos encontrados para juvenis de tambaqui, Colossoma macropomum (Façanha e Gomes, 2005) e de pacu, Piaractus mesopotamicus (Gonçalves et al., 2008) anestesiados com mentol. De acordo com os referidos autores, a concentração indicada para indução à anestesia profunda das espécies supracitadas é de $100 \mathrm{mg} \mathrm{L}^{-1}$ com tempos de indução e recuperação de $155,0 \pm 15,0$ e $302,0 \pm 40,0$ s e $102,3 \pm$ 29,5 e 113,2 $\pm 13,2 \mathrm{~s}$, para tambaqui e pacu, respectivamente. Estudo realizado com pintados, Pseudoplatystoma corruscans recomenda a concentração de $50 \mathrm{mg} \mathrm{L}^{-1}$ de mentol para produzir efeito de anestesia profunda (Vidal et al., 2006).

A indução a anestesia cirúrgica dos juvenis expostos à concentração de $60 \mathrm{mg} \mathrm{L}^{-1}$ foi obtida em tempo médio de $436,7 \pm 29,2 \mathrm{~s}$, tempo significativamente maior que o obtido com peixes expostos às concentrações de 120, 180, 240 e $300 \mathrm{mg} \mathrm{L}^{-1}$. Estas concentrações induziram os juvenis à anestesia cirúrgica em tempos estatisticamente semelhantes. Estes resultados sugerem a concentração de $120 \mathrm{mg} \mathrm{L}^{-1}$ como indicada para indução à anestesia cirúrgica, tendo em vista a questão econômica que, de acordo com Marking e Meyer (1985), é um dos pré-requisitos 
importantes durante a escolha de um anestésico. Esta concentração induziu os indivíduos ao referido estágio em tempo médio de $313,7 \pm 14,7 \mathrm{~s}$, tempo superior ao recomendado por Ross e Ross para indução anestésica de peixes. No entanto, Simões e Gomes (2009), utilizando mentol como anestésico, para juvenis de tilápia, obtiveram tempo mediano para indução a anestesia cirúrgica acima de 500 segundos quando submeteram os animais a concentração considerada adequada, $250 \mathrm{mg} \mathrm{L}^{-1}$. A diferença verificada no presente estudo pode estar relacionada à pureza do produto utilizado, uma vez que o autor supracitado necessitou de uma maior concentração de anestésico para obter o mesmo estágio de anestesia.

A recuperação dos indivíduos submetidos à concentração de $60 \mathrm{mg} \mathrm{L}^{-}$ 1 foi semelhante à obtida com animais expostos a $120 \mathrm{mg} \mathrm{L}^{-1}$ que por sua vez não apresentou diferença significativa em relação às concentrações de 180 e $240 \mathrm{mg} \mathrm{L}^{-1}$. A maior concentração (300 $m g \mathrm{~L}^{-1}$ ) foi também a que forneceu maior tempo de recuperação, 406,2 \pm 24,9 s. Este valor foi inferior ao tempo de recuperação obtido por Simões e Gomes (2009) em exposição de juvenis de tilápia do Nilo durante 10 minutos a concentração de $250 \mathrm{mg} \mathrm{L}^{-1}$ de mentol.

Para os adultos, os resultados de indução e recuperação para os diferentes estágios são mostrados na tabela 4. As menores concentrações (30, 60 e $\left.120 \mathrm{mg} \mathrm{L}^{-1}\right)$ não induziram os indivíduos ao estágio de anestesia profunda, confirmando que, para a tilápia do Nilo, a resistência ao anestésico utilizado eleva-se com o aumento do tamanho dos espécimes.
Tabela 4 - Eventos comportamentais (em segundos) de adultos de tilápia do Nilo, expostos a diferentes concentrações de mentol (1)

\begin{tabular}{|c|c|c|c|c|c|c|}
\hline \multirow{2}{*}{$\begin{array}{l}\text { Concen } \\
\text { tração } \\
\left(\mathrm{mg} \mathrm{L}^{-1}\right)\end{array}$} & \multicolumn{6}{|c|}{ Evento comportamental (segundos) } \\
\hline & $\begin{array}{l}\text { Está } \\
\text { gio I }\end{array}$ & $\begin{array}{l}\text { Estági } \\
\text { o II }\end{array}$ & $\begin{array}{l}\text { Estági } \\
\text { o III }\end{array}$ & $\begin{array}{l}\text { Estági } \\
\text { o IV }\end{array}$ & $\begin{array}{l}\text { Estági } \\
\text { o V }\end{array}$ & $\begin{array}{l}\text { Recup } \\
\text { eraçã } \\
\text { o }\end{array}$ \\
\hline & $\begin{array}{l}142, \\
7 \quad \pm\end{array}$ & & & & & \\
\hline 30 & $\begin{array}{l}10,0^{\mathrm{a}} \\
56,2 \\
\pm\end{array}$ & * & $\begin{array}{l}\text { * } \\
215,1 \\
\pm\end{array}$ & * & * & * \\
\hline 60 & & $\begin{array}{l}121,9 \\
\pm 14,4^{\mathrm{a}} \\
112,5 \\
\pm\end{array}$ & $\begin{array}{l} \pm \\
22,1^{\mathrm{a}} \\
184,2 \\
\pm\end{array}$ & * & * & * \\
\hline 120 & $\begin{array}{l}4,3^{b} \\
36,1\end{array}$ & $13,8^{\mathrm{a}, \mathrm{b}}$ & $\begin{array}{l}21,4^{a} \\
120,8\end{array}$ & 291,9 & * & * \\
\hline 180 & $\begin{array}{l} \pm \\
2,7^{\mathrm{b}, \mathrm{c}} \\
27,0\end{array}$ & $\begin{array}{l}75,0 \pm \\
5,9^{b, c} \pm\end{array}$ & $\frac{ \pm}{12,3^{b}}$ & $\begin{array}{l} \pm \\
38,6^{\mathrm{a}} \\
210,5\end{array}$ & $384,1^{*}$ & $\begin{array}{l}246,6 \\
\pm 13,0^{a}\end{array}$ \\
\hline 240 & $\begin{array}{l} \pm \\
1,5^{c} \\
21,2\end{array}$ & $\begin{array}{l}52,2 \\
3,3^{\mathrm{c}}\end{array}$ & $\begin{array}{l}91,9 \pm \\
6,2^{\mathrm{b}}\end{array}$ & $\begin{array}{l} \pm \\
17,3^{b} \\
151,3\end{array}$ & $\begin{array}{l} \pm \\
23,9 \mathrm{a} \\
320,1\end{array}$ & $\begin{array}{l}328,6 \\
\pm 32,0^{\mathrm{a}}\end{array}$ \\
\hline 300 & $\begin{array}{l} \pm \\
1,8^{c}\end{array}$ & $\begin{array}{l}39,9 \\
3,2^{\mathrm{c}}\end{array}$ & $\begin{array}{l}63,7 \pm \\
4,5^{b}\end{array}$ & $\begin{array}{l} \pm \\
12,2^{b}\end{array}$ & $\begin{array}{l} \pm \\
16,2^{b}\end{array}$ & $\begin{array}{l}247,4 \\
\pm 17,9^{\mathrm{a}}\end{array}$ \\
\hline
\end{tabular}

* Estágio não alcançado. (1) Médias seguidas pela mesma letra em coluna não diferem entre si pelo teste de Tukey $(P>0.05)$.

Iversen et al. (2003) ratificam que fatores biológicos tais como estágios de ciclo de vida e idade, tamanho e peso, teor de lipídios e sanidade precisam ser considerados durante a utilização de anestésicos. A concentração de $30 \mathrm{mg}$ $\mathrm{L}^{-1}$ de mentol induziu a tilápia adulta somente ao estágio de sedação leve, enquanto que as concentrações de 60 e $120 \mathrm{mg} \mathrm{L}^{-1}$ possibilitaram a indução até o estágio de narcose, em tempos estatisticamente semelhantes. Para estas três concentrações, a recuperação dos animais foi imediata após sua exposição à água sem anestésico.

As maiores concentrações (180, 240 e $300 \mathrm{mg} \mathrm{L}^{-1}$ ) induziram ao estágio de anestesia profunda, ideal para manejos rápidos e biometrias. Os tempos de indução a este estágio foram significativamente semelhantes para as duas últimas concentrações, que por sua vez foram estatisticamente diferentes da concentração de $180 \mathrm{mg}$ $\mathrm{L}^{-1}$. As concentrações de 240 e $300 \mathrm{mg}$ $\mathrm{L}^{-1}$ também permitiram aos peixes alcançarem 0 estágio de anestesia cirúrgica em tempos significativamente 
diferentes e recuperação em tempos estatisticamente semelhantes. Entretanto, os referidos tempos de indução, são superiores aos recomendados por Bell (1987). Para manejos rápidos e biometrias, a concentração recomendada é de 240 $\mathrm{mg} \mathrm{L}^{-1}$, pois a mesma permite a indução e recuperação em tempos de $210,5 \pm$ 17,3 s e $328,6 \pm 32,0$ s, respectivamente.

Em contrapartida, Anderson et al., (1997) encontraram tempos de indução e recuperação diferentes para adultos de truta arco-íris utilizando óleo de cravo como anestésico. Resultados diferentes também foram registrados para tambaqui anestesiado com mentol (Façanha e Gomes, 2005). Sabe-se que a resistência e a tolerância aos diferentes anestésicos variam de indivíduo para indivíduo e entre espécies (Hikasa et al., 1986), enquanto que a utilização de concentrações anestésicas determinadas para uma espécie pode levar a resultados perigosos para uma outra espécie (Tsantilas et al., 2006). Os mesmos autores afirmam que relação peso corpóreo/área da superfície branquial está diretamente relacionada à concentração anestésica exigida. De acordo com Ross e Ross (2008) existe uma relação inversa entre a taxa metabólica e peso corpóreo. Desta forma peixes menores e mais ativos exigirão concentrações inferiores quando comparados a espécimes maiores. Portanto a determinação de dosagens recomendadas para cada espécie e classe de tamanho é fundamental para procedimentos seguros durante a utilização de uma substância anestésica.

\section{CONCLUSÃO}

A utilização do mentol como anestésico mostrou-se eficaz, variando entre as diferentes classes de tamanho de tilápia do Nilo testadas. As concentrações recomendadas para anestesia profunda, ideal para manejos rápidos e biometrias, são de 60,180 , $240 \mathrm{mg} \mathrm{L}-1$, para alevinos, juvenis e adultos, respectivamente. Estudos posteriores deverão ser realizados com a finalidade de determinar a concentração recomendada para indução à anestesia cirúrgica em adultos, além de determinar o nível de estresse para as diferentes classes de tamanho.

\section{AGRADECIMENTOS}

À Coordenação de Aperfeiçoamento de Pessoal de Nível Superior - CAPES, pelo apoio financeiro. À Fundação Cearense de apoio e Desenvolvimento Científico (FUNCAP), ao Centro de Pesquisa em Aqüicultura Rodolpho von lhering do Departamento Nacional de Obras Contra as Secas/DNOCS e a GUABI NUTRIÇÃO ANIMAL pelo fornecimento de insumos oriundos da parceria firmada com nossa instituição de pesquisa.

\section{REFERÊNCIAS}

ANDERSON, G. W.; MCKINLEY, S. R.; COLAVECCHIA, M. The use of clove oil as an anesthetic for rainbow trout and its effects on swimming performance. North American Journal of Fisheries Management, v. 17, n. 2, p. 301-307, 1997.

BARBOSA, L. G.; MORAES, G.; INOUE, L. A. K. A. Respostas metabólicas do matrinxã submetido a banhos anestésicos de eugenol. Acta Scientiarum. Biological Science, v. 29, n. 3, p. 255-260, 2007.

BELL, G. An outline of anesthetic and anesthesia for salmonids, a guide for fish culturists in British Columbia. Canadian Technical Reporto of Fisheries and Aquatic Sciences. No.1534. 1987. 
FAÇANHA, M. F; GOMES, L. C. A eficácia do mentol como anestésico para tambaqui (Colossoma macropomum, Characiformes: Characidae). Acta Amazonica, v. 35, n. 1, p. 71-75, 2005.

FAO. Food and Agriculture Organization of the United Nations. Fish Stats Plus: universal software for fishery statistical time series: version 2.32. Rome, Italy, 2007.

GONÇALVES, A. F. N.; SANTOS, E. C. C.; FERNANDES, J. B. K.; TAKAHASHI, L. S. mentol e eugenol como substitutos da benzocaína na indução anestésica de juvenis de pacu. Acta Scientiarum. Animal Sciences, v. 30, n. 3, p. 339-344, 2008.

HIKASA, Y.; TAKASE, K.; OGASAWARA, T.; OGASAWARA, S. Anesthesia and recovery with tricaine methanesulfonate, eugenol and thiopental sodium in the carp. Cyprinus Carpio. Nippon Juigaku Zasshi, Towada, v.48, p.341351, 1986.

INOUE, L. A. K. A. SANTOS NETO, C. dos; MORAES, G. Clove oil as anaesthetic for juveniles of matrinxã Brycon cephalus (Gunther, 1869). Ciência Rural, v.33, n. 5, p. 943-947, 2003.

IVERSEN, M.; FINSTAD, B.; MCKINLEY, R. S.; ELIASSEN, R. A. The efficacy of metomidate, clove oil, Aqui-Sk and BenzoakR as anaesthetics in Atlantic salmon (Salmo salar L.) smolts, and their potential stress-reducing capacity. Aquaculture, v. 221, n. 1-4, p. 549566, 2003.

KING, W.V.; HOOPER, B.; HILLSGROVE, S.; BENTON, C.; BERLINSKY, D. L.; The use of clove oil, metomidate, tricaine methanesulphonate and 2-phenoxyethanol for inducing anaesthesia and their effect on the cortisol stress response in black sea bass (Centropristis striata L.). Aquaculture Research, v.36, n. 14, p.1442-1449, 2005.

KUBITZA, F. Tilápia: tecnologia e planejamento na produção comercial. Jundiaí: edição do autor, 2000. 285p.
LORENZO, D.; PAZ, D.; DELLACASSA, E.; DAVIES, P.; CANIGUERAL, S. Essential oils of Mentha pulegion and Mentha rotundifolia from Uruguay. Brazilian Archives of Biology and Technology, v. 45, p. 1-6, 2002.

MARKING, L.L.; MEYER, F.P. Are better anesthetics needed in fisheries. Fisheries, v.10, p.2-5, 1985.

MOREIRA, A. G. L.; TEIXEIRA, E. G.; CARREIRO, C. R. P.; MOREIRA, R. L. Eficácia do eugenol extraído da planta Eugenia aromatica como anestésico para realização de biometrias em adultos de tilápia do Nilo (Oreochromis niloticus). Acta Scientiarum. Animal Science, v. 32, n. 4, p. $419-423,2010$.

MYLONAS, C. C.; CARDINALETTI, G.; SIGELAKI, I.; POLZONETTI-MAGNI, A. Comparative efficacy of clove oil and 2phenoxyethanol as anesthetics in the aquaculture of European sea bass (Dicentrarchus labrax) and gilthead sea bream (Sparus aurata) at different temperatures. Aquaculture, v. 246, n. 1-4, p. 467-481, 2005.

PARK, I, S.; JO J. H.; LEE, S. J.; KIM, Y. A.; PARK, K. E.; HUR, J. W.; YOO, J. S.; SONG, Y. C. Anesthetic effect of lidocaine hydrochloridesodium bicarbonate and MS-222 on the greenling (Hexagrammos otakii). Journal of the Korean Fisheries Society v. 36 , p. 449-453, 2003.

PARK, M. O.; HUR, W. J.; IM, S.Y.; SEOL, D. W.; LEE, J.; PARK, I S. Anaesthetic efficacy and physiological responses to clove oilanaesthetized kelp grouper Epinephelus bruneus. Aquaculture Research, v. 39, n. 8, p. 877-884, 2008.

PATEL, T.; ISHIUJI, Y.; YOSIPOVITCH, G. Menthol: A refreshing look this ancient compound. American Academy of Dermatology, v. 57, p. 873-878, 2007.

ROSS, L.G.; ROSS, B. Anaesthetic and sedative techniques for aquatic animals. 3rd ed. Oxford: Blackwell Science, 2008. 236p. 
ROUBACH, R.; GOMES, L. C.; FONSECA, F. A. L.; VAL, A. L. Eugenol as an efficacious anaesthetic for tambaqui, Colossoma macropomum (Cuvier). Aquaculture Research, v. 36, n. 11, p. 1056-1061, 2005.

RUPPERT, E. E.; BARNES, R. D. 1994. Zoologia dos invertebrados. 6. Ed. Ed. Roca, São Paulo, 1994. 1056p.

SIMÕES, L. N.; GOMES, L. C. Eficácia do mentol como anestésico para juvenis de tilápiado-nilo (Oreochromis niloticus). Arquivo Brasileiro de Medicina Veterinária e Zootecnia, v. 61, n. 3, p.613-620, 2009.

TSANTILAS, $\quad H_{.} ; \quad$ GALATOS, A. D.; ATHANASSOPOULOU, F.; PRASSINOS, N. N. KOUSOULAKI, K. Efficacy of 2-phenoxyethanol as an anaesthetic for two size classes of white sea bream, Diplodus sargus L., and sharp snout sea bream, Diplodus puntazzo C. Aquaculture, v. 253, n. 1-4, p. 64-70, 2006.

VIDAL, L. V. O.; ALBINATI, R. C. B.; ALBINATI, A. C. L.; MACÊDO, G. R. de. Utilização do eugenol como anestésico para o manejo de juvenis de Pintado (Pseudoplatystoma corruscans). Acta Scientiarum. Biological Science. v. 28, n. 3, p. 275-279, 2006.

VIDAL, L. V. O.; ALBINATI, R. C. B.; ALBINATI, A. C. L.; LIRA, A. D. de; ALMEIDA, T. R. de; SANTOS, G. B. Eugenol como anestésico para a tilápia-do-nilo. Pesquisa Agropecuária Brasileira. v.43, n. 8, p.1069-1074, 2008.

VINATEA, L. A. Princípios químicos da qualidade da água em aqüicultura: uma revisão para peixes e camarões. 1. ed. Florianópolis: UFSC, 1997, $166 \mathrm{p}$.

WEBER, R. A.; PELETEIRO, J. B.; GARCIA MARTIN, L. O.; ALDEGUNDE, M. The efficacy of 2-phenoxyethanol, metomidate, clove oil and MS-222 as anaesthetic agents in the Senegalese sole (Solea senegalensis Kaup 1858). Aquaculture, v. 288, n. 1-2, p. 147-150, 2009.

ZAHL, I. H.; KIESSLING, A.; SAMUELSEN, O. B.; HANSEN, M. K. Anaesthesia of Atlantic cod
(Gadus morhua) - effect of pre-anaesthetic sedation, and importance of body weight, temperature and stress. Aquaculture, v. 295, n. 1-2, p. 52-59, 2009. 